\title{
Plasma Neutrophil Gelatinase-Associated Lipocalin as a Predictor of Renal Parenchymal Involvement in Infants With Febrile Urinary Tract Infection: A Preliminary Study
}

\author{
Bo Ae Yun, M.D., Eun Mi Yang (iD, M.D., and Chan Jong Kim (10, M.D. \\ Department of Pediatrics, Chonnam National University Hospital and Medical School, Gwangju, Korea
}

Background: Urinary tract infection (UTI) is the most common bacterial infection in infants. Renal parenchymal involvement is an important prognostic factor; however, early detection of parenchymal involvement in UTI may be difficult during infancy. This study aimed to assess whether a recently established biomarker of UTI, neutrophil gelatinaseassociated lipocalin (NGAL), can serve as a useful marker for the detection of cortical defects (CD) and to determine the appropriate diagnostic cut-off value of NGAL in infants with febrile UTI.

Methods: Infants hospitalized for febrile UTI were divided into two groups according to the presence of cortical defects on dimercaptosuccinic acid (DMSA) scintigraphy. Among 64 enrolled infants, 43 (67\%) had CD (UTI-CD) and 21 (33\%) had no CD (UTI-ND). The white blood cell count, C-reactive protein, and plasma NGAL (pNGAL) levels were determined before antibiotic therapy and compared between the two groups.

Results: pNGAL level was significantly higher in the UTI-CD group than in the UTI-ND group (340 $\mu \mathrm{g} / \mathrm{L}$ vs $214 \mu \mathrm{g} / \mathrm{L}, P=0.002$ ). Multivariate analysis showed that $\mathrm{pNGAL}$ level was the only independent predictor of $\mathrm{CD}$ (odds ratio 2.759, $P=0.039$ ). In the ROC curve analysis, pNGAL showed the highest area under the curve $(0.745 ; 95 \%$ confidence interval, 0.561-0.821; $P=0.014$ ). The appropriate cut-off value of pNGAL was $267 \mu \mathrm{g} / \mathrm{L}$ (sensitivity, $72.1 \%$; specificity, $71.4 \%$ ).

Conclusions: pNGAL was found to be a useful marker for early prediction of renal parenchymal involvement in infants with febrile UTI.

Key Words: Neutrophil gelatinase-associated lipocalin, Urinary tract infection, Cortical defect, Infant

\author{
Received: November 15, 2017 \\ Revision received: January 26, 2018 \\ Accepted: May 4, 2018 \\ Corresponding author: Eun Mi Yang \\ (i) https://orcid.org/0000-0001-9410-5855 \\ Department of Pediatrics, Chonnam \\ National University Hospital, 42 Jebong-ro, \\ Dong-gu, Gwangju 61469, Korea \\ Tel: +82-62-220-6647 \\ Fax: +82-62-222-6103 \\ E-mail: emyang@chonnam.ac.kr
}

Co-corresponding author: Chan Jong Kim (iD https://orcid.org/0000-0001-6519-2427 Department of Pediatrics, Chonnam National University Hospital and Medical School, 42 Jebong-ro, Dong-gu, Gwangju 61469, Korea

Tel: +82-62-220-6647

Fax: +82-62-222-6103

E-mail: cjkim@chonnam.ac.kr

\begin{abstract}
(C) Korean Society for Laboratory Medicine This is an Open Access article distributed under the terms of the Creative Commons Attribution Non-Commercial License (http://creativecommons.org/licenses/by-nc/4.0) which permits unrestricted non-commercial use, distribution, and reproduction in any medium, provided the original work is properly cited.
\end{abstract}

\section{INTRODUCTION}

Urinary tract infection (UTI) is a common bacterial infection in infants, with an incidence of $2 \%$ during the first two years of life [1]. Infants with UTI are more likely than the older children to have significant morbidity and acute pyelonephritis (APN). Infants have non-specific symptoms such as fever and irritability and are less able to communicate their symptoms, leading to potential delays in diagnosis. Infants have a higher incidence of sequelae after APN than older children, suggesting an age-related risk [2, 3]. Thus, early and accurate detection of APN is a primary goal, especially in infants with febrile UTI.

In clinical practice, symptoms along with acute-phase inflammatory markers such as white blood cell (WBC) count, erythrocyte sedimentation rate, C-reactive protein (CRP), and procalcitonin have been used to detect cortical defects $[4,5]$. However, 
when used alone, these inflammatory markers may not accurately reflect cortical defects (CD) owing to their non-specific nature [6]. Tc-99m dimercaptosuccinic acid (DMSA) renal scintigraphy is considered optimal for the detection of renal parenchymal involvement or scarring in UTI [7]. However, this invasive method requires sedation and exposure to radiation. Therefore, a more practical method is needed to determine renal parenchymal involvement.

Evolving biomarkers have been studied for non-invasive detection of APN in children with UTI [8-11]. Although several studies have revealed promising results for neutrophil gelatinase-associated lipocalin (NGAL) in children with UTI [9-11], only limited data are available for infants [12, 13]. This study aimed to assess whether plasma NGAL (pNGAL) could be used as a reliable marker for renal parenchymal involvement and to determine the appropriate diagnostic cut-off value of NGAL for detection of renal parenchymal involvement in infants with febrile UTI.

\section{METHODS}

\section{Study design and infant selection}

This retrospective study was conducted at a single tertiary center (Chonnam National University Hospital, Gwangju, Korea) between January 2015 and July 2016. Hospitalized infants under 12 months of age and who had febrile UTI were enrolled. Exclusion criteria were as follows: (1) underlying disease, (2) previous febrile UTI, and (3) delayed blood sampling after administration of antibiotics. UTI was defined as an abnormal urinalysis result and a positive urine culture ( $\geq 5 \times 10^{4}$ colony forming units $/ \mathrm{mL}$ ) according to the American Academy of Pediatrics guidelines [14]. The infants were divided into two groups based on the presence or absence of CD on DMSA scintigraphy. Of the 64 infants enrolled, 43 (67.2\%) had CD (UTI-CD) and 21 (32.8\%) had no CD (UTI-ND). The median age was 4.0 months; 41 were males and 23 were females. Medical records were reviewed for data such as age, sex, urine culture results, and laboratory and radiological parameters. The study was approved by the Institutional Review Board of Chonnam National University Hospital (CNUH-2017-124), Gwangju, Korea and followed the Declaration of Helsinki.

\section{Laboratory and radiological investigations}

Blood samples for WBC, CRP, and PNGAL and urine samples were obtained on admission before third generation cephalosporin therapy. Blood ( $5 \mathrm{~mL}$ ) was collected by vein puncture in
Vacutainer K3 EDTA Tubes (Becton-Dickinson and Co., Franklin Lakes, NJ, USA) and Vacutainer Plus Plastic Serum Tubes. WBC count was determined with UniCel DxH 800 (Beckman Coulter, Brea, CA) using the impedance method, and immunoturbidimetric assay of CRP was carried out with Olympus AU5800 autoanalyzer (Olympus Co., Tokyo, Japan). pNGAL concentration was measured using a commercial kit (in 2015, Triage NGAL test; Alere, San Diego, CA, USA; since 2016, NGAL ELISA kit; BioPorto Diagnostics, Gentofte, Denmark). NGAL concentrations were expressed as $\mathrm{ng} / \mathrm{mL}$. The measurable range of NGAL is $15-1,300 \mathrm{ng} / \mathrm{mL}$ by Triage NGAL test and $25-3,000 \mathrm{ng} / \mathrm{mL}$ by NGAL ELISA kit. Inter- and intra-assay CVs of the assays were, $13.5 \%$ and $11 \%$ for the Triage test, respectively, and $5 \%$ for the NGAL ELISA kit. Urine (approximately $10 \mathrm{~mL}$ ) was collected using a catheter, and automated dipstick urinalysis was carried out (CLINITEK Novus Analyzer, Siemens, Erlangen, Germany). All infants were evaluated by ultrasound (US) and DMSA scintigraphy to identify any anatomical abnormalities and the presence of renal parenchymal involvement, respectively. DMSA scintigraphy was performed 2-3 hours following the IV injection of 99m-technetium-DMSA (Tecnescan, Mallinckrodt Diagnostica, Netherlands) using a dose of $50 \mu \mathrm{Ci}(1.85 \mathrm{MBq}) / \mathrm{kg}$ (minimum dose $500 \mu \mathrm{Ci}$ ). DMSA scintigraphy was repeated six months later in children with renal CD. Voiding cystourethrography was conducted in children with any abnormal findings on US or DMSA scintigraphy to check the vesicoureteral reflux. The severity of vesicoureteral reflux was classified according to the International Reflux Study Committee classification [15].

\section{Statistical analysis}

Categorical data were summarized as counts and percentages, and continuous data were summarized as median values with interquartile range (IQR). Continuous data were analyzed using the Student's t-test or Mann-Whitney $U$ test as appropriate, and categorical data were analyzed using the chi-square test. Logistic regression was used to assess predictors of $C D$. As NGAL and CRP data were not normally distributed, log-transformed data was used for logistic regression. In the primary analysis, univariate analysis was used to identify the association between $C D$ and the following factors: age, sex, hydronephrosis, WBC count, MPV, log CRP, and log pNGAL concentrations. We included all significant variables in a multivariate model. ROC curve analysis was performed, and the area under the curve (AUC) was calculated to identify the best predictive cut-off values for WBC, CRP, and pNGAL for the detection of CD. The sensitivity and specificity for the best cut-off values were calculated. $P<0.05$ was con- 
sidered statistically significant. All analyses were performed with SPSS ver. 21.0 (SPSS Inc., Chicago, IL, USA).

\section{RESULTS}

\section{Baseline and clinical characteristics}

The characteristics and laboratory data of the UTI-CD and UTIND groups are summarized in Table 1. There were no significant differences in sex, median age, duration of fever, and US abnormalities between the groups (Table 1). A total of 38 infants underwent VCUG (33 in UTI-CD and five in UTI-ND). Ten (30.3\%) of the infants in the UTI-CD group and two (40\%) of the infants in the UTI-ND group had vesicoureteral reflux, but this percentage did not significantly differ between the two groups (Table 1).

\section{Biomarkers}

WBC, CRP, and pNGAL values were significantly higher in the UTI-CD group than in the UTI-ND group. Univariate analysis showed that elevated WBC count $(P=0.049)$, log CRP level

Table 1. Characteristics and laboratory data of infants with UTI

\begin{tabular}{|c|c|c|c|c|}
\hline Variables & Total $(N=64)$ & UTI-CD (N=43) & UTI-ND (N=21) & $P^{*}$ \\
\hline Age (month), median (IQR) & $4.0(2.0-5.0)$ & $3.0(2.0-5.0)$ & $4.0(3.5-5.5)$ & 0.206 \\
\hline Sex (male/female) & $41 / 23$ & $31 / 12$ & $10 / 11$ & 0.055 \\
\hline $\begin{array}{l}\text { Fever duration (day), median (IC } \\
\text { Total } \\
\text { Before initiating antibiotics }\end{array}$ & $\begin{array}{l}2(1.3-3) \\
1(1-2)\end{array}$ & $\begin{array}{l}2(2-3) \\
1(1-2)\end{array}$ & $\begin{array}{l}2(1-3) \\
2(1-2.5)\end{array}$ & $\begin{array}{l}0.921 \\
0.548\end{array}$ \\
\hline $\begin{array}{l}\text { Laboratory findings, median (IQ } \\
\text { WBC }\left(10^{\circ} / \mathrm{L}\right) \\
\text { Hb }(\mathrm{g} / \mathrm{L}) \\
\mathrm{PLT}\left(10^{9} / \mathrm{L}\right) \\
\mathrm{MPV}(\mathrm{fL}) \\
\mathrm{CRP}(\mathrm{nmol} / \mathrm{L}) \\
\text { pNGAL }(\mu \mathrm{g} / \mathrm{L}) \\
\text { pNGAL WBC }\end{array}$ & $\begin{array}{c}15.3(10.4-18.9) \\
112(104-120) \\
377(316-452) \\
8.9(8.6-9.5) \\
31.4(19.0-54.3) \\
285(174-413) \\
0.019(0.012-0.030)\end{array}$ & $\begin{array}{c}15.5(10.8-19.4) \\
110(103-119) \\
397(337-457) \\
8.8(8.4-9.2) \\
33.3(23.8-73.3) \\
340(203-465) \\
0.024(0.013-0.033)\end{array}$ & $\begin{array}{c}11.7(10.7-16.6) \\
117(107-121) \\
357(300-415) \\
9.1(8.6-9.7) \\
22.7(12.4-38.1) \\
214(105-285) \\
0.016(0.008-0.026)\end{array}$ & $\begin{array}{l}0.042 \\
0.162 \\
0.137 \\
0.135 \\
0.014 \\
0.002 \\
0.010\end{array}$ \\
\hline $\begin{array}{l}\text { Imaging findings } \\
\text { Abnormal US' }(\mathrm{N}, \%)\end{array}$ & $19(29.7 \%)$ & 15 (35.7\%) & $4(19.0 \%)$ & 0.174 \\
\hline
\end{tabular}

Categorical data (sex) were analyzed using the chi-square test, and continuous data were analyzed using Student's t-test. Continuous data not normally distributed (CRP and NGAL) were analyzed using the Mann-Whitney $U$ test.

${ }^{*}$ Compared with the UTI-CD group and UTI-ND group; ${ }^{\dagger}$ Abnormal US includes hydronephrosis, renal pelviectasis, and abnormal parenchymal echogenicity. Abbreviations: UTI, urinary tract infection; UTI-CD, urinary tract infection with cortical defect; UTI-ND, urinary tract infection without cortical defect; IQR, interquartile range; WBC, white blood cells; PLT, platelet; MPV, mean platelet volume; CRP, C-reactive protein; pNGAL, plasma neutrophil gelatinase-associated lipocalin; US, ultrasonography.

Table 2. Logistic regression analyses to identify predictors of cortical defects in infants with febrile urinary tract infection

\begin{tabular}{|c|c|c|c|c|}
\hline \multirow{2}{*}{ Variables } & \multicolumn{2}{|c|}{ Univariate } & \multicolumn{2}{|c|}{ Multivariate } \\
\hline & OR (95\% Cl) & $P$ & OR (95\% Cl) & $P$ \\
\hline Age & $0.857(0.675-1.088)$ & 0.205 & & \\
\hline Sex (male) & $2.842(0.119-1.042)$ & 0.059 & & \\
\hline WBC $\left(10^{9} / L\right)$ & $1.0(1-1)$ & 0.049 & $1.0(1-1)$ & 0.213 \\
\hline MPV (fL) & $0.584(0.288-1.187)$ & 0.137 & & \\
\hline $\log$ CRP (nmol/L) & $2.247(1.106-4.563)$ & 0.025 & $1.398(0.608-3.213)$ & 0.430 \\
\hline $\log$ pNGAL $(\mu \mathrm{g} / \mathrm{L})$ & $3.531(1.476-8.450)$ & 0.005 & $2.759(1.051-7.240)$ & 0.039 \\
\hline Hydronephrosis & $2.361(0.671-8.314)$ & 0.181 & & \\
\hline
\end{tabular}

Abbreviations: WBC, white blood cells; log CRP, log transformed C-reactive protein; MPV, mean platelet volume; log NGAL, log transformed plasma neutrophil gelatinase-associated lipocalin; $\mathrm{OR}$, odds ratio; $\mathrm{Cl}$, confidence interval. 


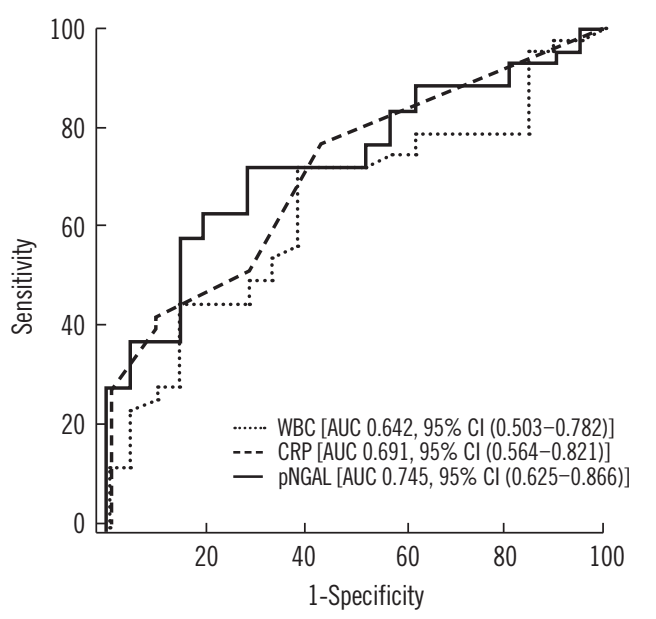

\begin{tabular}{lccccc}
\hline Variables & $\begin{array}{c}\text { Cut-off } \\
\text { levels* }\end{array}$ & $\begin{array}{c}\text { Sensitivity } \\
\left(\%^{\dagger}, 95 \% \mathrm{Cl}\right)\end{array}$ & $\begin{array}{c}\text { Specificity } \\
\left(\%^{\dagger}, 95 \% \mathrm{Cl}\right)\end{array}$ & $\begin{array}{c}\text { PPV } \\
\left(\%^{\dagger}, 95 \% \mathrm{Cl}\right)\end{array}$ & $\begin{array}{c}\text { NPV } \\
\left(\%^{\dagger}, 95 \% \mathrm{Cl}\right)\end{array}$ \\
\hline WBC $\left(10^{9} / \mathrm{L}\right)$ & 12.7 & $72.1(56.3-84.7)$ & $61.9(38.4-81.9)$ & $79.5(68.5-87.3)$ & $52(37.6-66.1)$ \\
CRP $(\mathrm{nmol} / \mathrm{L})$ & 30.5 & $60.5(44.4-75.0)$ & $66.7(43.0-85.4)$ & $78.8(65.9-87.7)$ & $45.2(33.8-57.0)$ \\
pNGAL $(\mu \mathrm{\mu g} / \mathrm{L})$ & 267 & $72.1(56.3-84.7)$ & $71.4(47.8-88.7)$ & $83.8(71.9-91.2)$ & $55.6(41.9-68.4)$ \\
\hline
\end{tabular}

Fig. 1. ROC curve analysis for the detection of cortical defects in infants with febrile urinary tract infection.

*The cut-off point selection in the context of ROC curve analysis is the maximum of the Youden index with the level of confidence set at 95\%; 'Two-by-two tables were used to calculate sensitivity, specificity, PPV, and NPV for cut-off value of variables and the level of confidence set at $95 \%$.

Abbreviations: WBC, white blood cells; CRP, C-reactive protein; pNGAL, plasma neutrophil gelatinase-associated lipocalin; AUC, area under curve; $\mathrm{Cl}$, confidence interval; PPV, positive predictive value; NPV, negative predictive value.

$(P=0.025)$, and log pNGAL concentration $(P=0.005)$ were related to the presence of $C D$ (Table 2). Multivariate analysis showed that log pNGAL concentration was the only independent predictor of $\mathrm{CD}$ (odds ratio [OR] 2.759, $P=0.039$ ). ROC curve analysis showed that PNGAL had the highest AUC of $0.745(95 \% \mathrm{Cl}, 0.561-0.821, P=0.014)$, followed by 0.691 for CRP $(95 \% \mathrm{Cl}, 0.561-0.821, P=0.014)$, and 0.642 for WBC (95\% Cl, 0.503-0.782, $P=0.066$ ) (Fig. 1). pNGAL showed fair predictive accuracy for $\mathrm{CD}$. According to the ROC curve analysis, the appropriate cut-off values for WBC, CRP and pNGAL were $12.7 \times 10^{9} / \mathrm{L}, 30.5 \mathrm{nmol} / \mathrm{L}$, and $267 \mu \mathrm{g} / \mathrm{L}$, respectively. $\mathrm{pN}$ GAL showed the highest sensitivity (72.1\%), specificity (71.4\%), positive predictive value (PPV) $(83.8 \%)$, and negative predictive value (NPV) (55.6\%) for CD (Fig. 1 and Table 2).

\section{DISCUSSION}

The present study revealed that pNGAL concentrations were significantly higher in UTI-CD group than in UTI-ND group. Compared with WBC count and CRP, pNGAL had the highest AUC and the highest sensitivity, specificity, PPV, and NPV. pNGAL concentration was the only independent predictor of $C D$. These results suggest that pNGAL could be a useful marker for the detection of CD in infants with febrile UTI.

UTI is a common bacterial infection in infants and children, with approximately $1 \%$ of boys and $1-3 \%$ of girls developing a UTI during childhood [16]. APN, which refers to infection of the renal parenchyma, may cause chronic renal damage. Renal scarring may lead to hypertension, renal insufficiency, and endstage renal failure. In clinical practice, some inflammatory markers have been used to detect infection of the renal parenchyma, but these markers may not accurately reflect the $\operatorname{CD}[6,16]$.

According to several clinical studies, NGAL is an excellent marker for the detection and monitoring of acute kidney injury [17] and is an emerging biomarker for the diagnosis of UTI [12, 18]. Recently, NGAL has been proposed as a marker of CD in UTI [11]. Several studies have analyzed data on children, but limited data are available for infants [13], and the diagnostic value of NGAL for the prediction of CD in infants with UTI is unclear. Two pediatric UTI studies reported optimal cut-off levels for pNGAL to be $102.5 \mu \mathrm{g} / \mathrm{L}$ [11] and $117 \mu \mathrm{g} / \mathrm{L}$, respectively [19], for the prediction of APN. In our study, the optimal cut-off level was $267 \mu \mathrm{g} / \mathrm{L}$ in infants with febrile UTI. Furthermore, the UTI-ND group also showed high levels of pNGAL (mean: 204.9 $\mu \mathrm{g} / \mathrm{L}$ ) compared with pediatric UTI groups in previous studies $[11,19]$. No international studies have been conducted to date to accurately evaluate the reference values of NGAL measurement in blood or urine samples based on large population surveys, stratified according to age, gender, and ethnicity [20]. Stejskal et al [21] reported a median pNGAL concentration of 78.8 $\mathrm{ng} / \mathrm{mL}$ in men and of $80.0 \mathrm{ng} / \mathrm{mL}$ in women in healthy white individuals [21]. The pNGAL levels for the detection of acute renal injury in adult patients range from 150 to $400 \mathrm{ng} / \mathrm{mL}$ [22]. However, there is no established reference value for NGAL in infants. 
According to a previous study, the distribution of urine NGAL (uNGAL) values in pediatric-age patients approximates a lognormal distribution, with values being higher in neonates than in children [23]. During acute bacterial infection, the optimal cut-off value of pNGAL was $217 \mu \mathrm{g} / \mathrm{L}$ in older children and 247 $\mu \mathrm{g} / \mathrm{L}$ in young children (below the age of two years) [24]. Of note, in this study, high concentrations of pNGAL were observed in infants compared with children with UTI. The reason for this observation remains unknown. Previously reported cut-off values for NGAL did not allow accurate prediction of the presence of parenchymal involvement in infants with febrile UTI in this study (using a cut-off of $102.5 \mu \mathrm{g} / \mathrm{L}$ [11]: sensitivity, $93 \%$ and specificity, 24\%; and a cut-off of $117 \mu \mathrm{g} / \mathrm{L}$ [19]: sensitivity, $88 \%$ and specificity, 38\%). On the other hand, our cut-off value for NGAL $(267 \mu \mathrm{g} / \mathrm{L})$ showed a sensitivity of $72 \%$ and a specificity of $71.4 \%$ in detecting $C D$.

NGAL can be tested in both blood and urine. These two forms of NGAL have distinct mechanisms of induction and upregulation, allowing for different applications in clinical settings [9]. pNGAL has been reported to be a useful predictor of systemic inflammation associated with pyelonephritis and damage [11, $13,19]$, while UNGAL is also a potentially useful marker for the detection of subclinical renal damage such as scarring, vesicoureteral reflux, or obstruction [9, 25-27]. In UTI, NGAL production by neutrophils may increase the actual NGAL concentration. As NGAL may be released from neutrophils during the preparation of serum, it may be preferable to use plasma [20]. In the present study, we used plasma samples and corrected the PNGAL values to the WBC counts. Our study revealed the potential of pNGAL as a sensitive and useful marker for early prediction of parenchymal involvement.

Our study has some limitations. First, given the relatively small sample size, our results should be interpreted with caution. Second, pNGAL was measured only at admission, thus, time-series data were not available. Third, the assay method for NGAL measurement was changed from the Triage NGAL test to the ELISA kit, and the assay method may affect the measured levels; however, no significant differences were observed in our study. Fourth, the cut-off value in our study was higher than that used in previous studies. This may be due to differences in enrollment criteria; further studies should be based on a larger population.

In conclusion, rapid screening of pNGAL at admission provides valuable information for the detection of parenchymal involvement for the prediction of the clinical course in infants with acute febrile UTI.

\section{Authors' Disclosures of Potential Conflicts of Interest}

The authors declare that they have no conflicts of interest.

\section{Acknowledgments}

We thank all our colleagues for excellent help.

\section{REFERENCES}

1. Jakobsson B, Esbjörner E, Hansson S. Minimum incidence and diagnostic rate of first urinary tract infection. Pediatrics 1999;104:222-6.

2. Peters CA, Skoog SJ, Arant BS Jr., Copp HL, Elder JS, Hudson RG, et al. Summary of the AUA guideline on management of primary vesicoureteral reflux in children. J Urol 2010;184:1134-44.

3. Park YS. Renal scar formation after urinary tract infection in children. Korean J Pediatr 2012;55:367-70.

4. Jaye DL and Waites KB. Clinical applications of C-reactive protein in pediatrics. Pediatr Infect Dis J 1997;16:735-46.

5. de Man P. Bacterial attachment, inflammation and renal scarring in urinary tract infection. Wien Med Wochenschr 1991;141:537-40.

6. Mori R, Yonemoto N, Fitzgerald A, Tullus K, Verrier-Jones K, Lakhanpaul M. Diagnostic performance of urine dipstick testing in children with suspected UTI: a systematic review of relationship with age and comparison with microscopy. Acta Paediatr 2010;99:581-4.

7. Goldraich NP and Goldraich IH. Update on dimercaptosuccinic acid renal scanning in children with urinary tract infection. Pediatr Nephrol 1995;9:221-6.

8. Lee HE, Kim DK, Kang HK, Park K. The diagnosis of febrile urinary tract infection in children may be facilitated by urinary biomarkers. Pediatr Nephrol 2015;30:123-30.

9. Forster CS and Devarajan P. Neutrophil gelatinase-associated lipocalin: utility in urologic conditions. Pediatr Nephrol 2017;32:377-81.

10. Yim HE, Yim H, Bae ES, Woo SU, Yoo KH. Predictive value of urinary and serum biomarkers in young children with febrile urinary tract infections. Pediatr Nephrol 2014;29:2181-9.

11. Sim JH, Yim HE, Choi BM, Lee JH, Yoo KH. Plasma neutrophil gelatinase-associated lipocalin predicts acute pyelonephritis in children with urinary tract infections. Pediatr Res 2015;78:48-55.

12. Valdimarsson S, Jodal U, Barregård L, Hansson S. Urine neutrophil gelatinase-associated lipocalin and other biomarkers in infants with urinary tract infection and in febrile controls. Pediatr Nephrol 2017;32:2079-87.

13. Seo WH, Nam SW, Lee EH, Je BK, Yim HE, Choi BM. A rapid plasma neutrophil gelatinase-associated lipocalin assay for diagnosis of acute pyelonephritis in infants with acute febrile urinary tract infections: a preliminary study. Eur J Pediatr 2014;173:229-32.

14. Subcommittee on Urinary Tract Infection, Steering Committee on Quality Improvement and Management, Roberts KB. Urinary tract infection: clinical practice guideline for the diagnosis and management of the initial UTI in febrile infants and children 2 to 24 months. Pediatrics 2011;128:595-610.

15. International Reflux Study Committee. Medical versus surgical treatment of primary vesicoureteral reflux: report of the International Reflux Study Committee. Pediatrics. 1981;67:392-400.

16. Elder JS. Urinary tract infections. In: Kliegman RM, ed. Nelson textbook 
of pediatrics. 19th ed. Philadelphia: Elsevier Saunders, 2011:1829-34.

17. Haase M, Bellomo R, Devarajan P, Schlattmann P, Haase-Fielitz A, NGAL Meta-analysis Investigator Group. Accuracy of neutrophil gelatinase-associated lipocalin (NGAL) in diagnosis and prognosis in acute kidney injury: a systematic review and meta-analysis. Am J Kidney Dis 2009;54:1012-24.

18. Kim BH, Yu N, Kim HR, Yun KW, Lim IS, Kim TH, et al. Evaluation of the optimal neutrophil gelatinase-associated lipocalin value as a screening biomarker for urinary tract infections in children. Ann Lab Med 2014;34:354-9.

19. Kim BK, Yim HE, Yoo KH. Plasma neutrophil gelatinase-associated lipocalin: a marker of acute pyelonephritis in children. Pediatr Nephrol 2017;32:477-84.

20. Clerico A, Galli C, Fortunato A, Ronco C. Neutrophil gelatinase-associated lipocalin (NGAL) as biomarker of acute kidney injury: a review of the laboratory characteristics and clinical evidences. Clin Chem Lab Med 2012;50:1505-17.

21. Stejskal D, Karpísek M, Humenanska V, Hanulova Z, Stejskal P, Kusnierova P, et al. Lipocalin-2: development, analytical characterization, and clinical testing of a new ELISA. Horm Metab Res. 2008;40:381-5.

22. Di Somma S, Magrini L, De Berardinis B, Marino R, Ferri E, Moscatelli P, et al. Additive value of blood neutrophil gelatinase-associated lipocalin to clinical judgement in acute kidney injury diagnosis and mortality prediction in patients hospitalized from the emergency department. Crit Care 2013;17:R29.

23. Cangemi G, Storti S, Cantinotti M, Fortunato A, Emdin M, Bruschettini M, et al. Reference values for urinary neutrophil gelatinase-associated lipocalin (NGAL) in pediatric age measured with a fully automated chemiluminescent platform. Clin Chem Lab Med 2013;51:1101-5.

24. Fjaertoft G, Foucard T, Xu S, Venge P. Human neutrophil lipocalin (HNL) as a diagnostic tool in children with acute infections: a study of the kinetics. Acta Paediatr 2005;94:661-6.

25. Rafiei A, Mohammadjafari H, Bazi S, Mirabi AM. Urinary neutrophil gelatinase-associated lipocalin (NGAL) might be an independent marker for anticipating scar formation in children with acute pyelonephritis. J Renal Inj Prev 2015;4:39-44.

26. Parmaksiz G, Noyan A, Dursun H, Ince E, Anarat R, Cengiz N. Role of new biomarkers for predicting renal scarring in vesicoureteral reflux: NGAL, KIM-1, and L-FABP. Pediatr Nephrol 2016;31:97-103.

27. Noyan A, Parmaksiz G, Dursun H, Ezer SS, Anarat R, Cengiz N. Urinary NGAL, KIM-1 and L-FABP concentrations in antenatal hydronephrosis. J Pediatr Urol 2015;11:249. 